\title{
SEMIAMARTS AND FINITE VALUES
}

\author{
BY ULRICH KRENGEL AND LOUIS SUCHESTON
}

Communicated by Alexandra Bellow, February 9, 1977

Let $X_{n}$ be a sequence of real-valued random variables adapted to an in. creasing sequence of $\sigma$-algebras $F_{n}$. We denote by $T, T_{f}, \bar{T}$ respectively the collection of bounded, finite, and arbitrary stopping times for $\left(F_{n}\right)_{n \in N}$. This paper reports on recent progress concerning the theory of semiamarts, i.e. processes for which $\left(E X_{\tau}\right)_{\tau \in T}$ is bounded, initiated in [3], and the theory of amarts, i.e. processes for which $\lim _{\tau \in T} E X_{\tau}$ exists. We relate the notion of semiamart to processes of interest in the theory of optimal stopping (cf. [2]), namely $X_{n}$ such that $\left|E X_{\mu}\right|<\infty$ for $\mu \in T_{f}$, or for $\mu \in \bar{T}$. For independent random variables $X_{n}$ and for processes of the form $X_{n}=c_{n}^{-1} \sum_{i=1}^{n} Y_{i}$ with increasing $c_{n}$ 's and independent nonnegative $Y_{i}$ 's, a new dominated estimate

$$
E\left(\sup X_{n}^{+}\right) \leqslant K \sup _{\mu \in \bar{T}} E X_{\mu} \quad(=K V(\bar{T}))
$$

with $K=2$ in the first and $K<5.46$ in the second case, shows that such process. es are semiamarts if and only if $\sup \left|X_{n}\right|$ is integrable. Also in the case when $F_{n}=F_{m}$ for all $n, m \in \mathbf{N}$, a semiamart has a necessarily integrable supremum. This observation is used to construct averages of aperiodic stationary sequences, which are not semiamarts-thereby strengthening a result announced by A. Bellow [1]. This can be done also in the "descending" case, i.e. when the time domain $\mathbf{N}$ is replaced by $\mathbf{- N}$ (see [3]); thus our results indicate that there are no connections between the amart theory and the ergodic theory of point transformations.

THEOREM 1 (RIESZ DECOMPOSITION FOR SEMIAMARTS). Every semiamart $\left(X_{n}, F_{n}\right)$ can be represented as $X_{n}=Y_{n}+Z_{n}$ where $\left(Y_{n}, F_{n}\right)$ is a martingale and $\left(Z_{n}, F_{n}\right)$ is an $L_{1}$-bounded semiamart such that for each $A \in \bigcup F_{m}$

$$
\underset{n}{\lim \inf } \frac{1}{n} \sum_{i=1}^{n} \int_{A} Z_{i} \leqslant 0 \leqslant \lim _{n} \sup \frac{1}{n} \sum_{i=1}^{n} \int_{A} Z_{i}
$$

This generalizes the Riesz decomposition for amarts [3]. A variant of Theorem 1 permits us to give necessary and sufficient conditions for the uniqueness of the Riesz decomposition. One consequence of the Riesz decomposition is:

$A M S$ (MOS) subject classifications (1970). Primary 60G40, $60 \mathrm{G} 45$.

1 The research of this author is in part supported by the National Science Foundation. Copyright $\odot 1977$, American Mathematical Society 
THEOREM 2. Let $X_{n}$ be a semiamart (amart) such that for some $\alpha \geqslant 1$ $\sum_{i=1}^{\infty} i^{-(1+\alpha)} E\left|X_{i}-X_{i-1}\right|^{2 \alpha}<\infty ;$ then sup $\left|X_{n}\right| / n<\infty$ a.s. (resp. $X_{n} / n \rightarrow$ 0 a.s.).

Theorem 2 extends the strong law of large numbers for martingale differences; a somewhat weaker version of this, and of the next theorem, appears in [3].

THEOREM 3 (AMART OPTIONAL SAMPLING THEOREM). Let $\mu_{n} \in T_{f}, \mu_{1} \leqslant$ $\mu_{2} \leqslant \mu_{3} \leqslant \cdots$. Let $X_{n}$ be an amart, $\hat{X}_{n}=X_{\mu_{n}}$ and assume

(a) $E\left|\hat{X}_{n}\right|<\infty \forall n \in \mathbf{N}$ and

(b) $\lim _{N \rightarrow \infty} \int_{\left\{\mu_{n}>N\right\}}\left|X_{N}\right|=0 \forall n \in \mathbf{N}$.

Then $\left(\hat{X}_{n}, G_{n}\right)$ is an amart where $G_{n}=F_{\mu_{n}}=\left\{A \in F: A \cap\left\{\mu_{n}=k\right\} \in\right.$ $\left.F_{k} \forall k\right\}$. If also $\mu_{n} \rightarrow \infty$ then the Riesz decomposition of $\hat{X}_{n}$ has the martingale part $Y_{n}=Y_{\mu_{n}}$ and the potential part $\hat{Z}_{n}=Z_{\mu_{n}}$, where $Y_{n}+Z_{n}$ is the amart Riesz decomposition of $X_{n}$.

THEOREM 4. There exists a semiamart which converges a.s. and in $L_{1}$ but is not an, amart.

There exist two simple methods of construction of amarts and semiamarts: (1) each adapted sequence $X_{n}$ is a semiamart if $\sup \left|X_{n}\right| \in L_{1}$. Such a sequence is an amart iff in addition $X_{n}$ converges a.s.; (2) quasimartingales are amarts.

THEOREM 5. In general a semiamart or amart cannot be decomposed into two summands arising from constructions (1) and (2). In fact, there exists a nonnegative predictable amart which is a potential (the martingale part in its Riesz decomposition vanishes), with $\sup _{n} E\left(X_{n} \log ^{+} X_{n}\right) \leqslant 1$ and $E \sup X_{n}=\infty$.

THEOREM 6. Let $\left(X_{n}\right)_{n \in \mathrm{N}}$ be a sequence of adapted random variables for the increasing sequence $\left(F_{n}\right)_{n \in N}$, with $\sup E\left|X_{n}\right|=M<\infty .\left(X_{n}\right)$ is a semiamart iff for each $\nu \in \bar{T}$ such that $E\left(1_{\{\nu<\infty\}} X_{\nu}\right)$ is defined as an extended real number, one has $\left|E\left(1_{\{\nu<\infty\}} X_{\nu}\right)\right|<\infty$. If the $\sigma$-algebra $F_{\infty}$ generated by all $F_{n}$ 's is nonatomic, a further equivalent condition is: for each $\nu \in T_{f}$ such that $E X_{\nu}$ is defined as an extended real number, one has $\left|E X_{\nu}\right|<\infty$.

If $\left(E X_{\tau}\right)_{\tau \in T}$ is unbounded from above one can find $\nu$ with $E X_{\nu}^{-}<\infty$ and $E X_{\nu}^{+}=\infty$. Thus the theorem can be interpreted as saying that for $L_{1}$-bounded processes with infinite value $V(T)=\sup _{\tau \in T} E X_{\tau}$, the value $V(\bar{T})$ is assumed, and $V\left(T_{f}\right)$ is assumed if $F_{\infty}$ is nonatomic. In the descending case $V\left(T_{f}\right)=\infty$ is assumed if $\left(X_{n}\right)$ is $L_{1}$-bounded or each $F_{-n}$ is nonatomic. Since then $V\left(T_{f}\right)=\infty$ is equivalent to $V(T)=\infty$, this yields an analogous characterization of descending semiamarts.

THEOREM 7. If $\left(X_{n}\right)$ is adapted to $\left(F_{n}\right)$ and $X_{n+1}$ is independent of $F_{n}$ for all $n$, then $E \sup X_{n}^{+} \leqslant 2 V(\bar{T})$.

We only showed the existence of a constant $K_{0}$ such that $2 \leqslant K_{0} \leqslant 4$, 
and $E \sup X_{n}^{+} \leqslant K_{0} V(\bar{T})$. That $K_{0}$ may be chosen equal to 2 is due to D. Garling.

Now let $\left(Y_{n}\right)$ be adapted to the increasing family $\left(F_{n}\right)$ and assume that $Y_{n+1}$ is independent of $F_{n}$ for all $n$. Call $\left(X_{n}\right)$ a sequence of averages of nonnegative independent random variables if $X_{n}$ is of the form $X_{n}=c_{n}^{-1} \sum_{i=1}^{n} Y_{i}$ with $1 \leqslant c_{1}<c_{2}<\cdots$.

THEOREM 8. If $\left(X_{n}\right)$ is a sequence of averages of nonnegative independent random variables then $E\left(\sup X_{n}\right)<5.46$ where $V=V(\bar{T})=V\left(T_{f}\right)=V(T)$.

This result has an interesting probabilistic interpretation. If $X_{n}$ is the fortune of a player at time $n$, then $V$ is the maximal expected gain of a player $A$ using nonanticipating stopping rules. $E$ sup $X_{n}$ equals $\sup _{\mu} E X_{\mu}$ where the supremum is over all measurable random variables $\mu: \Omega \rightarrow \mathrm{N}$. Thus $E \sup X_{n}$ is the maximal expected gain of a player $B$ endowed with complete foresight. The theorem may be interpreted as saying that, whatever be the sequence of distributions, the odds 5.46:1 are favorable to $A$ even against an omniscient opponent $B$ playing the same game.

A consequence of Theorem 9 is that a sequence of averages of nonnegative independent random variables is a semiamart for $\left(F_{n}\right)$ iff $\sup X_{n} \in L_{1}$.

Call a point-transformation $S$ aperiodic if there exists no measurable $B$ with $P(B)>0$ such that for some $n \in \mathrm{N}$ and all measurable $A \subset B$, the symmetric differences $A \Delta S^{-n} A$ has measure 0. The result of A. Bellow [1] is strengthened by

THEOREM 9. If $S$ is an aperiodic invertible measure preserving transformation of $(\Omega, F, P)$ then there exists an $f \in L_{1}^{+}$for which $X_{n}=n^{-1} \sum_{k=0}^{n-1} f \circ S^{k}$ is not a semiamart, ascending or descending.

\section{REFERENCES}

1. A. Bellow, Stability properties of the class of asymptotic martingales, Bull. Amer. Math. Soc. 82 (1976), 338-340.

2. Y. S. Chow, H. Robbins and D. Siegmund, Great expectations: the theory of optimal stopping, Houghton Mifflin, Boston, Mass., 1971. MR 48 \#10007.

3. G. A. Edgar and L. Sucheston, Amarts: $A$ class of asymptotic martingales. $A$. Discrete parameter, J. Multivariate Analysis 6 (1976), 193-221.

INSTITUTE FOR MATHEMATICAL STATISTICS, UNIVERSITY OF GOTTINGEN, GOTTINGEN, FEDERAL REPUBLIC OF GERMANY OHIO 43210

DEPARTMENT OF MATHEMATICS, OHIO STATE UNIVERSITY, COLUMBUS, 